\title{
Global shadowing of pseudo-Anosov homeomorphisms
}

\author{
MICHAEL HANDEL \\ Department of Mathematics, Columbia University, New York, NY 10027, USA
}

(Received 10 January 1985)

Abstract. We prove that if $f: M^{2} \rightarrow M^{2}$ is pseudo-Anosov and if $g \simeq f$, then there is a closed subset $X \subset M^{2}$ and a continuous surjection $\pi: X \rightarrow M$ that is homotopic to inclusion such that $f \circ \pi=\pi \circ g \mid X$.

\section{Introduction}

In this paper we consider A. B. Katok's notion of $K$-global shadowing (defined in $\S 1$ ) as it applies to a pseudo-Anosov homeomorphism $f: M^{2} \rightarrow M^{2}$ on a closed surface. It is an equivalence relation which allows one to compare the orbits of $f$ with the orbits of any map $g: M^{2} \rightarrow M^{2}$ that is homotopic to $f$. We write $(f, x) \sim^{K}(g, y)$, or $(f, x) \sim(g, y)$ when $K$ is unspecified.

Global shadowing in a pseudo-Anosov homotopy class generalizes two other well known and useful equivalence relations:

(A) When $x$ and $y$ are fixed points of $f^{n}$ and $g^{n}$ respectively $(n>0)$, then $(f, x) \sim(g, y)$ if and only if $\left(f^{n}, x\right)$ is Nielsen equivalent to $\left(g^{n}, y\right)$.

(B) When $K=\varepsilon$ is sufficiently small and $g$ is $\varepsilon$-close to $f$ in the $C^{0}$-topology, then $(f, x) \sim(g, y)$ if and only if the $f$-orbit of $x \varepsilon$-shadows (in the sense of [B]) the pseudo-orbit of $f$ defined by the $g$-orbit of $y$. Lewowicz [L] has considered $\varepsilon$ shadowing in the pseudo-Anosov context.

Thurston showed ([T]; Lemma 2.1 below) that from the point of view of Nielsen equivalence, $f$ has the minimal number of periodic points among all maps in its homotopy class. Our first theorem shows that from the point of view of global shadowing, $f$ has the minimal number of orbits among all maps in its homotopy class.

THeOREM 1. Let $f: M^{2} \rightarrow M^{2}$ be a pseudo-Anosov homeomorphism on a closed surface, and let $\mathrm{g}: \mathrm{M}^{2} \rightarrow M^{2}$ be any map that is homotopic to $f$. Then

(i) $\left(f, x_{1}\right) \sim\left(f, x_{2}\right) \Rightarrow x_{1}=x_{2}$;

(ii) for all $x \in M^{2}$, there exists $y \in M^{2}$ such that $(f, x) \sim(g, y)$; if $x$ is $f$-periodic with least period $n$, then $y$ can be chosen to be g-periodic with least period $n$.

Our second theorem is a uniformization of theorem 1. When $f$ is Anosov rather than pseudo-Anosov, then theorem 2 reduces to the fact (proposition 2.1 of [F]) 
that any map that is homotopic to an Anosov diffeomorphism is semi-conjugate (by a map which is homotopic to the identity) to that Anosov diffeomorphism.

THEOREM 2. Let $f: M^{2} \rightarrow M^{2}$ be a pseudo-Anosov homeomorphism of a closed surface and let $\mathrm{g}: \mathrm{M}^{2} \rightarrow M^{2}$ be any map that is homotopic to $f$. Then there exists a closed set $Y \subset M^{2}$ and a surjective map $\varphi: Y \rightarrow M$ which is homotopic to the inclusion map such that $f \varphi=\varphi g \mid Y$.

Theorem 1 is in response to a question of A. B. Katok. I am grateful to him for bringing it to my attention. I would also like to thank P. Boyland for several interesting conversations which provoked theorem 2 .

This research was supported in part by NSF Grant No. MCS8120790.

\section{Notation and definitions}

For the remainder of the paper, $f: M^{2} \rightarrow M^{2}$ will be a pseudo-Anosov homeomorphism of a closed surface and $g: M^{2} \rightarrow M^{2}$ will be a map that is homotopic to $f$. The reader that is not familiar with pseudo-Anosov homeomorphisms should consult [T] and [F-L-P] as required. We will use only the following properties of $f: M^{2} \rightarrow M^{2}$.

(1.1) The periodic points of $f$ are dense.

(1.2) The action induced by $f$ on the free homotopy classes of $M$ has no periodic orbits.

(1.3) The fixed point index of a fixed point $x$ of $f^{n}$ is never 0 .

(1.4) There exist $\lambda>1$ and an equivariant metric $\tilde{D}$ on the universal cover $\tilde{M}$ of $M$ such that $\tilde{D}=\sqrt{\tilde{D}_{s}^{2}+\tilde{D}_{u}^{2}}$, where $\tilde{D}_{s}: \tilde{M} \times \tilde{M} \rightarrow[0, \infty)$ and $\tilde{D}_{u}: \tilde{M} \times \tilde{M} \rightarrow[0, \infty)$ are equivariant functions satisfying:

$$
\tilde{D}_{u}\left(\tilde{f} \tilde{x}_{1}, \tilde{f} \tilde{x}_{2}\right)=\lambda \tilde{D}_{u}\left(\tilde{x}_{1}, \tilde{x}_{2}\right) \quad \text { and } \quad \tilde{D}_{s}\left(\tilde{f}^{-1} \tilde{x}_{1}, \tilde{f}^{-1} \tilde{x}_{2}\right)=\lambda \tilde{D}_{s}\left(\tilde{x}_{1}, \tilde{x}_{2}\right)
$$

for all $\tilde{x}_{1}, \tilde{x}_{2} \in \tilde{M}$ and all lifts $\tilde{f}$ of $f$.

Remark. The stable and unstable foliations for $f$ lift to stable and unstable foliations for $\tilde{f}$. Given $\tilde{x}_{1}, \tilde{x}_{2} \in \tilde{M}, \tilde{D}_{s}\left(\tilde{x}_{1}, \tilde{x}_{2}\right)$ is defined to be the minimum length, with respect to the transverse measure on the stable foliation of $\tilde{f}$, of an arc connecting $\tilde{x}_{1}$ to $\tilde{x}_{2}$. The function $\tilde{D}_{u}$ is defined similarly with respect to the unstable foliation.

We fix once and for all a lift $\tilde{f}: \tilde{M} \rightarrow \tilde{M}$ of $f$ to the universal cover $\tilde{M}$ of $M$. As $g$ is homotopic to $f$ there is a unique lift $\tilde{\mathrm{g}}: \tilde{M} \rightarrow \tilde{M}$ which is equivariantly homotopic to $\tilde{f}$.

Definition 1.5 (A. B. Katok). The $f$-orbit of $x$ is $K$-globally shadowed by the $g$-orbit of $y$ if there are lifts $\tilde{x}$ of $x$ and $\tilde{y}$ of $y$ such that $\tilde{D}\left(\tilde{f}^{k}(\tilde{x}), \tilde{g}^{k}(\tilde{y})\right) \leq K$ for all $k \in \mathbb{Z}$. We write $(f, x) \sim^{K}(g, y)$ or $(f, x) \sim(g, y)$ if the shadowing constant $K$ is unspecified. Remark. The equivalence classes $(f, x) \sim(g, y)$ are not dependent on the choice of equivariant metric in the definition of global shadowing. We use $\tilde{D}$ because it is convenient for computations.

Definition 1.6. If $x$ is a fixed point of $f^{n}$ and $\tilde{x}$ is a lift of $x$, then $\tilde{f}^{n}(\tilde{x})=s \tilde{x}$ for some covering translation $s$ of $\tilde{M}$. Similarly, if $y$ is a fixed point of $g^{n}$ and $\tilde{y}$ is a 
lift of $y$, then $\tilde{g}^{n}(\tilde{y})=t \tilde{y}$ for some covering translation $t$. We say that $\left(f^{n}, x\right)$ and $\left(g^{n}, y\right)$ are Nielsen equivalent if there exist $\tilde{x}$ and $\tilde{y}$ such that $s=t$.

The following lemma establishes relationship (A) stated in the introduction.

LeMmA 1.7. If $x$ is a fixed point of $f^{n}$ and $y$ is a fixed point of $g^{n}$, then $\left(f^{n}, x\right)$ is Nielsen equivalent to $\left(g^{n}, y\right)$ if and only if $(f, x) \sim(g, y)$.

Proof. (only if) Suppose that $\tilde{f}^{n}(\tilde{x})=t \tilde{x}$ and $\tilde{g}^{n}(\tilde{y})=t \tilde{y}$. Then

$$
\begin{aligned}
\tilde{D}\left(\tilde{f}^{k}(\tilde{x}), \tilde{g}^{k}(\tilde{y})\right) & =\tilde{D}\left(\tilde{f}^{k-n}(t \tilde{x}), \tilde{g}^{k-n}(t \tilde{y})\right) \\
& =\tilde{D}\left(t^{\prime} \tilde{f}^{k-n}(\tilde{x}), t^{\prime} \tilde{g}^{k-n}(\tilde{y})\right)=\tilde{D}\left(\tilde{f}^{k-n}(\tilde{x}), \tilde{g}^{k-n}(\tilde{y})\right),
\end{aligned}
$$

since $t^{\prime}=\tilde{f}^{k-n} t \tilde{f}^{-(k-n)}=\tilde{g}^{k-n} t \tilde{g}^{-(k-n)}$ is a covering translation and $\tilde{D}$ is equivariant. Thus $\tilde{D}\left(\tilde{f}^{k}(\tilde{x}), \tilde{g}^{k}(\tilde{y})\right)$ takes on only finitely many values and is bounded.

(if) Suppose that $\tilde{f}^{n}(\tilde{x})=s \cdot \tilde{x}, \tilde{g}^{n}(\tilde{y})=t \cdot y$ and that $(f, x) \sim^{K}(g, y)$. Then

$$
\tilde{D}\left(\tilde{x},\left(s^{-1} \tilde{g}^{n}\right)^{k} \tilde{y}\right)=\tilde{D}\left(\left(s^{-1} \tilde{f}^{n}\right)^{k} \tilde{x},\left(s^{-1} \tilde{g}^{n}\right)^{k} \tilde{y}\right) \leq K \quad \text { all } k \in \mathbb{Z}
$$

Since any bounded subset of $\tilde{M}$ intersects only finitely many lifts of $y,\left(s^{-1} \tilde{g}^{n}\right)^{k}$ fixes both $\tilde{y}$ and $s^{-1} \tilde{g}^{n}(\tilde{y})=s^{-1} t \tilde{y}$ for some $k>0$. This implies that $\left(s^{-1} \tilde{g}^{n}\right)^{k}$ commutes with the covering translation $s^{-1} t$ and hence by property (1.2) that $s^{-1} t=$ identity.

\section{Proofs of theorems 1 and 2}

We begin this section with a proof of a result of Thurston which, in conjunction with lemma 1.7 , implies that $f$-periodic orbits of least period $n$ are globally shadowed by $g$-periodic orbits of least period $n$. The heart of this paper (lemma 2.2 ) is the observation that there is a uniform bound to the shadowing constants produced by lemma 2.1 .

LEMMA 2.1 [T]. (i) If $x_{1}$ and $x_{2}$ are distinct fixed points of $f^{n}$ then $\left(f^{n}, x_{1}\right)$ and $\left(f^{n}, x_{2}\right)$ are not Nielsen equivalent.

(ii) If $x$ is $f$-periodic with least period $n$, then there exists $y$ which is $g$-periodic with least period $n$ and such that $\left(f^{n}, x\right)$ is Nielsen equivalent to $\left(g^{n}, y\right)$.

Proof. Property (1.4) implies that no lift of any iterate of $f$ can fix two distinct points. This immediately implies (i). The existence of $y$ such that $\left(g^{n}, y\right)$ is Nielsen equivalent to $\left(f^{n}, x\right)$ is now a consequence of property (1.3) and [Br; theorem $3, \mathrm{p}$. 94]. It suffices to show that $y$ has least period $n$.

There exist lifts $\tilde{x}$ and $\tilde{y}$ and a covering translation $t$ such that $t^{-1} \tilde{f}^{n}(\tilde{x})=\tilde{x}$ and $t^{-1} \tilde{g}^{n}(\tilde{y})=\tilde{y}$. If $y$ has least period $m_{1}<n$, then (by the uniqueness of lift of $g^{n}$ fixing $\tilde{y})$ there is a covering translation $t_{1}$ such that $t^{-1} \tilde{g}^{n}=\left(t_{1} \tilde{g}^{m_{1}}\right)^{m_{2}}$ where $m_{2}=n / m_{1}>1$. Since equivariantly homotopic lifts of $f^{n}$ are equal,

$$
t^{-1} \tilde{f}^{n}=\left(t_{1} \tilde{f}^{m_{\mathrm{r}}}\right)^{m_{2}}
$$

This implies that the entire $t_{1} \tilde{f}^{m_{1}}$ orbit of $\tilde{x}$ is fixed by $t^{-1} \tilde{f}^{n}$ in contradiction to our observation above that no lift of an iterate of $f$ can fix two distinct points. 
LEMma 2.2. There exists $K=K(g)$ such that $(f, x) \sim(g, y)$ if and only if $(f, x) \sim^{K}(g, y)$. In particular, if $x_{n} \rightarrow x, y_{n} \rightarrow y$, and $\left(f, x_{n}\right) \sim\left(g, y_{n}\right)$ then $(f, x) \sim(g, y)$. Proof. Let $R=\max \left[\sup _{\tilde{x} \in \tilde{M}} \tilde{D}(\tilde{f}(\tilde{x}), \tilde{g}(\tilde{x})), \sup _{\tilde{x} \in \tilde{M}} \tilde{D}\left(\tilde{f}^{-1}(\tilde{x}), \tilde{g}^{-1}(\tilde{x})\right)\right]$. Note that by equivariance of $\tilde{D}$ and compactness of $M, R<\infty$. Property (1.4) implies that

$$
\tilde{D}_{u}(\tilde{f}(\tilde{x}), \tilde{g}(\tilde{y})) \geq \lambda \tilde{D}_{u}(\tilde{x}, \tilde{y})-R
$$

and

$$
\tilde{D}_{s}\left(\tilde{f}^{-1}(\tilde{x}), \tilde{g}^{-1}(\tilde{y})\right) \geq \lambda \tilde{D}_{s}(\tilde{x}, \tilde{y})-R .
$$

Let $K=2(R+1) /(\lambda-1)$. If $\tilde{D}_{u}(\tilde{x}, \tilde{y})>K / 2$, then $\tilde{D}_{u}(\tilde{f} \tilde{x}, \tilde{g} \tilde{y})>1+\tilde{D}_{u}(\tilde{x}, \tilde{y})$. Similarly, if $\tilde{D}_{s}(\tilde{x}, \tilde{y})>K / 2$, then $\tilde{D}_{s}\left(\tilde{f}^{-1}(\tilde{x}), \tilde{g}^{-1}(\tilde{y})\right) \geq 1+\tilde{D}_{s}(\tilde{x}, \tilde{y})$. It follows that if $(f, x) \sim(g, y)$ then $(f, x) \sim{ }^{\kappa}(g, y)$.

Suppose now that $\tilde{x}_{n} \rightarrow \tilde{x}, \tilde{y}_{n} \rightarrow \tilde{y}$ and that $\tilde{D}\left(\tilde{f}^{k} \tilde{x}_{n}, \tilde{g}^{k} \tilde{y}_{n}\right) \leq K$ for all $k$ and $n$. Since $\tilde{D}\left(\tilde{f}^{k} \tilde{x}, \tilde{g}^{k} \tilde{y}\right) \leq \tilde{D}\left(\tilde{f}^{k} \tilde{x}, \tilde{f}^{k} \tilde{x}_{n}\right)+\tilde{D}\left(\tilde{f}^{k} \tilde{x}_{n}, \tilde{g}^{k} \tilde{y}_{n}\right)+\tilde{D}\left(\tilde{g}^{k} y_{n}, \tilde{g}^{k} y\right)$ for all $n$, and the first and third terms in this sum tend to zero as $n$ tends to infinity,

$$
\tilde{D}\left(\tilde{f}^{k} \tilde{x}, \tilde{g}^{k} \tilde{y}\right) \leq \sup _{n \in \mathbb{Z}} \tilde{D}\left(\tilde{f}^{k} \tilde{x}_{n}, \tilde{g}^{k} \tilde{y}_{n}\right) \leq K .
$$

Thus $(f, x) \sim^{K}(g, y)$ as desired.

Proof of theorem 1. Part (i) follows immediately from property (1.4). Part (ii) follows from lemmas 1.7, 2.1, and 2.2 and from property (1.1).

Proof of theorem 2. Define $Y=\left\{y \in M^{2}: \exists x \in M^{2}\right.$ such that $\left.(f, x) \sim(g, y)\right\}$. Theorem 1 implies that for each $y_{0} \in Y$ there is a unique $x_{0} \in M^{2}$ such that $\left(f, x_{0}\right) \sim\left(g, y_{0}\right)$; define $\varphi: Y \rightarrow M$ by $\varphi\left(y_{0}\right)=x_{0}$. Lemma 2.2 implies that $Y$ is closed and $\varphi$ is continuous. By construction, $\tilde{\varphi}: \tilde{Y} \rightarrow \tilde{M}$ is a bounded distance from the inclusion. This implies that $\varphi$ is homotopic to the inclusion. Finally, since $(f, x) \sim(g, y)$ implies $(f, f(x)) \sim(g, g(y)), f \varphi=\varphi g \mid Y$.

Remark 2.3. The proof of theorem 2 carries over to the case that $M=T^{2}$ and $f$ is an Anosov diffeomorphism. Note that the usual $\varepsilon$-shadowing arguments (e.g. [B]) apply to $\tilde{f}: \mathbb{R}^{2} \rightarrow \mathbb{R}^{2}$ with no restriction on the size of $\varepsilon$. Since for each $\tilde{y} \in \mathbb{R}^{2}$ the $\tilde{g}$-orbit of $\tilde{y}$ is an $R$-pseudo-orbit for $\tilde{f}$, it is always possible to find $x \in T^{2}$ such that $(f, x) \sim(g, y)$. Thus the set $Y \subset T^{2}$ constructed in the proof of theorem 2 equals all of $T^{2}$ and $g$ is semi-conjugate to $f$ (cf. proposition 2.1 of [F]).

Remark 2.4. When $\partial M \neq 0, \tilde{D}$ is an incomplete metric on int $\tilde{M}$. In terms of the proofs, one thinks of $\tilde{D}$ as a complete metric on $\tilde{M}^{*}$ which is obtained from $\tilde{M}$ by collapsing each component of $\partial \tilde{M}$ to a point. The only change required in theorem 1 is that (i) should read: $\left(f, x_{1}\right) \sim\left(f, x_{2}\right) \Rightarrow x_{1}, x_{2}$ are in the same component of $\partial M$. In theorem 2 , change $M$ to int $M$ in the conclusions.

\section{REFERENCES}

[B] R. Bowen. On Axiom A diffeomorphisms. Regional conference series in mathematics; no. 35 (1978).

[Br] R. Brown. The Lefschetz Fixed Point Theorem. Scott Foresman, 1971.

[F-L-P] A. Fathi, F. Laudenbach \& V. Poénaru. Travaux de Thurston sur les surfaces. Asterisque 66-67 1979. 
[F] J. Franks. Anosov diffeomorphisms. Proceedings of the Symposium in Pure Mathematics, 14 pp. 61-94.

[L] J. Lewowicz. Persistence in expansive systems. Preprint.

[T] W. Thurston. On the geometry and dynamics of diffeomorphisms of surface. Preprint. 